\title{
EFFICIENT 3D DOCUMENTATION OF NEPTUNE FOUNTAIN IN THE PARK OF SCHÖNBRUNN PALACE AT MILLIMETER SCALE
}

\author{
P. Dorninger ${ }^{\mathrm{a}, *}$, C. Nothegger ${ }^{\mathrm{a}}$, S. Rasztovits ${ }^{\mathrm{b}}$ \\ a 4D-IT GmbH, Austria - (p.dorninger, c.nothegger)@4d-it.com \\ ${ }^{\mathrm{b}}$ Dept. of Geodesy and Geoinformation, TU Vienna, Austria - sascha.rasztovits@ geo.tuwien.ac.at
}

KEY WORDS: Terrestrial Laser Scanning, 3D-Modeling, Triangulation, Accuracy, Schloß Schönbrunn

\begin{abstract}
:
3D documentation of cultural heritage is commonly based on image sensors or laser scanning. Respective sensors and software tools have been improved significantly in recent years concerning both automation and accuracy. Considering the documentation of huge objects with high richness in detail requires a huge effort. Close-range scanners offer the highest accuracy, however, their restricted field-of-view and their limited maximum measurement distance make numerous scanning positions necessary, thus significantly increasing the effort for data acquisition and processing. This is similar if image processing is used for aiming at mm-accuracy. For this, several hundred images are typically necessary for modelling sculptures properly. Consequently, both methods are not applicable for huge objects with an extension of several tens of meters and with a highly structured surface. Within this contribution, we describe a workflow for data acquisition and processing of the Neptune Fountain in the park area of the UNESCO World Heritage Site Schloß Schönbrunn in Vienna, Austria. Some 5 billion points were acquired from 230 scanning positions within four days using a phase-shift laser scanner. Highly automated processing of this data resulted in a triangulation model with an absolute accuracy of less than $5 \mathrm{~mm}$ for non-translucent materials. Problems at the translucent marble structures are analysed and a textured $3 \mathrm{D}$ model of a portion of the object for the application on mobile devices is presented.
\end{abstract}

\section{INTRODUCTION}

3D documentation is essential for modern preservation of cultural heritage. Various digital data acquisition and processing technologies are commonly used. Concerning the sensor technology, the instruments can be assigned to two groups: image sensors (digital cameras) and laser scanning. For the generation of 3D models based on image data, a series of tools and software suites exists (e.g. Vergauwen \& Van Gool, 2006, Deseilligny \& Clery, 2011). In the past decade, laser scanning gained in importance concerning fast, automated and accurate acquisition of high resolution and highly detailed 3D models. An indisputable advantage of image based 3D modelling is its implicit capability for model texturing. However, the achievable accuracy and resolution of the 3D models is lower than those of laser scanning data (GolparvarFard et al., 2011).

To achieve mm-accuracy, several hundred images are necessary to reconstruct the shape of objects like sculptures (PierrotDeseilligny et al., 2011). So-called mid-range laser scanner using phase-shift distance measurement (e.g. Faro Focus, Leica HDS7000, Z+F Imager 5010) enable the acquisition of comparably huge parts of an object from a single scanning position achieving mm-accuracy as well. The highest accuracy may be achieved using close-range laser scanner. These instruments are typically based on profile or pattern projection (Akca, 2012). However, the application of close-range laser scanners for huge objects is a challenging task considering their restricted field-of-view and their low maximum scanning distance. Therefore, if huge objects are to be modelled, similar to image based acquisition configurations, numerous scanning positions are required.

Considering this, the application of image based modelling as well as close-range scanners become expensive in both, effort and costs, if huge objects (some $10 \mathrm{~m}$ in extension) are to be modelled at mm-accuracy. We investigated the applicability of phase-shift laser scanning for this task. In 2011, the Neptune Fountain in the park area of the UNESCO World Heritage Site Schloß Schönbrunn in Vienna, Austria, has been scanned using a Faro Photon 120 scanner (www.faro.com). In this contribution, we present the workflow developed for this task. The results are discussed, considering both economical aspects and the achievable accuracy.

\section{RELATED WORK}

For 3D documentation by terrestrial laser scanning (TLS) using a phase-shift scanner, the instrument is typically placed on numerous scanning positions. From each position, the object's surface is recorded as a pattern of individual measurement points, the point-cloud. To merge these point-clouds for subsequent model generation, a unique coordinate reference system has to be defined and the individual scans have to be transformed into this reference system. This registration process can be realized by locally minimizing the discrepancies between overlapping scans. Typically, a variant of the iterative closes point (ICP) approach is used for this (Rusinkiewicz \& Levoy, 2001, Segal et al., 2009). Alternatively, distinct reference points, either realized as signalized points or manually selected

\footnotetext{
* Corresponding author.
} 
within the point-clouds, can be used to determine the respective transformation matrices for the individual scans (Ullrich et al., 2003). An approach considering reference points for global accuracy while minimizing local discrepancies by ICP is described by Akca \& Gruen (2007).

The resulting point-cloud is unstructured, i.e. it does not contain any information about the topologically correct relations between points. Furthermore the point-cloud usually contains both noise and systematic errors. Therefore, the accurate and correct reconstruction of the originally sampled surface from TLS data is a challenging problem.

There are two basic approaches to surface reconstruction. One approach is to utilize geometric properties of the point-cloud. Examples of this approach include algorithms such as alpha shapes (Edelsbrunner \& Mücke, 1994), power crust (Amenta et al., 2001), or VRIP (Curless \& Levoy, 1996). Algorithms which try to construct highly generalized models by fitting geometric primitives also fall into this category. The common property is that an explicit description of the surface itself is constructed.

The alternative are implicit surfaces. Here the goal is to find a function defined on the entire volume around the object. The surface itself is then defined as an iso-contour of this scalar field. Examples of this approach include the implicit surface framework described in (Hoppe, 1995), and Poisson surfaces (Kazhdan et al., 2006, Bolitho et al., 2009, Kazhdan \& Hoppe, 2013). An advanced implementation of the latter was used for this work (Nothegger, 2011).

\section{EXPERIMENT}

\subsection{The Neptune Fountain in Schloß Schönbrunn}

The Neptune Fountain was designed as the crowning element of the Great Parterre of the park areal of Schloß Schönbrunn. The excavations for the main basin began in 1776 and the fountain was completed four years later under the commission of Maria Theresa.

The main basin has an extension of some 100 by $50 \mathrm{~m}$ (Fig. 1). The cascade basins are $35 \mathrm{~m}$ in width and the total height from the bottom of the basin to the top of the Neptune sculpture is approximately $23 \mathrm{~m}$ (Fig. 2). The sculpture of Neptune is placed in a shell-shaped chariot on top of a rocky grotto. Together with a nymph and the sea-goodness Thetis, it builds up the central sculpture ensemble on top of the cascade basins. Four additional sculpture ensembles representing Tritons, creatures who are half-man and half-fish, and belong to Neptune's entourage are placed on top of the cascade basins.

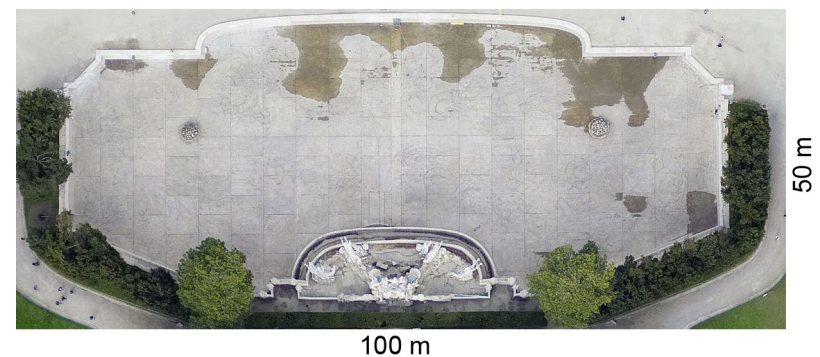

Figure 1. Ortho-image of the Neptune Fountain showing the main basin, the cascade basins, and the 5 sculpture ensembles.

\subsection{Data acquisition and point-cloud registration}

The private management of Schloß Schönbrunn decided to geometrically document their inventory at mm-accuracy. Those documentation models may serve as foundation for future reconstruction after partially or complete destruction i.e. by visitors. In order to achieve mm-accuracy with an economically justifiable effort, we decided to use a Faro Photon 120 phaseshift TLS. The scanning campaign was performed by $A V D$ Vermessung ZT GmbH, Dornbirn, Austria (www.avd-zt.at) using a Faro Photon 120 scanner. Within 4 days, some 5 billion points were measured at the entire object from 230 scanning positions. For the cascade basins and the sculpture ensembles, we aimed at achieving scanning distances of 2 to maximal 10 meter, resulting in an average point sampling distance of $3 \mathrm{~mm}$ per scan.

To maximize the coverage while minimizing shadowed regions, a complex set up planning was necessary. To achieve optimal viewing conditions, a movable but stable scaffold was used (Fig. 2) with a platform height of 8 meters. Figure 2 gives an impression of the dimensions of the object considering the size of the sitting operator (blue) and the silver tripod with the scanner both on top of the scaffold.

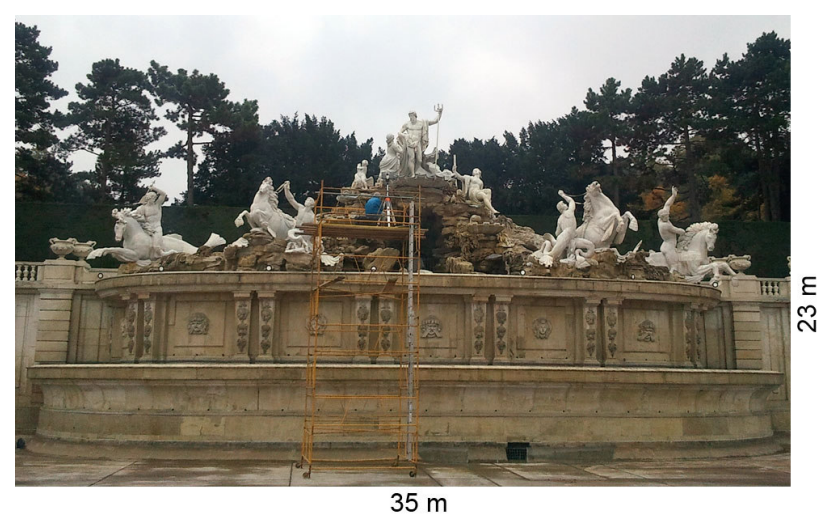

Figure 2. Scanning position on top of a scaffold in front of the cascade basins showing the extension of the Neptune Fountain.

Figure 3 shows an orthogonal projection of the reduced pointcloud. The colour coding represents the local height with respect to the reference height at the bottom of the main basin (blue $=0 \mathrm{~m}$ ) to the top of the Neptune sculpture (dark red $=23$ $\mathrm{m})$. The white regions (no points) are caused by remaining water in the basins as no points could be measured in these regions. As figure 3 shows an unfiltered point-cloud, the positions of the scaffold arranged around the lower cascade basin are visible in dark red.

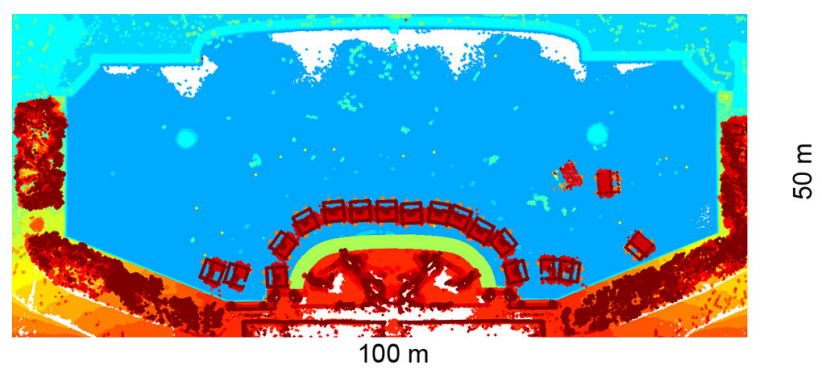

Figure 3. Height coded, reduced point-cloud of the entire scene.

The main basin (blue), the cascade basins and the sculpture ensembles (bottom) and the positions of the scaffold (dark red, arranged around the cascade basins) can be seen clearly. 
In order to ensure a high absolute accuracy for the entire object, control points defined by planar and spherical targets were used (Fig. 4). The positions of the control points were determined using a tachymeter.

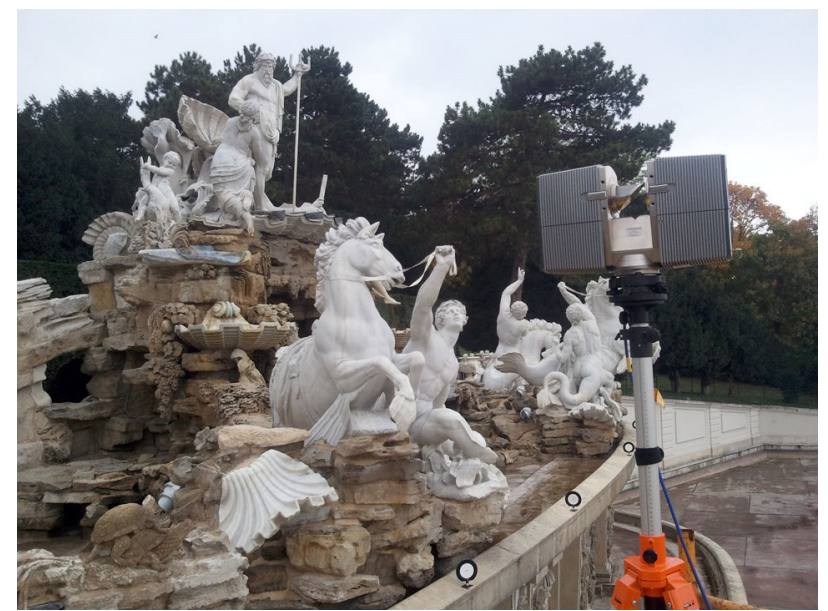

Figure 4. Scanning position on top of the scaffold and planar targets for registration arranged along the border of the cascade basin.

\subsection{D Modelling}

For 3D model generation, we used an advanced implementation of the Poisson triangulation described by Nothegger (2011). The automated processing chain requires individual pointclouds per scanning position. For the determination of the implicit surface (confer section 2), surface normal vectors (per point) with a known orientation with respect to the surface are necessary. The point-clouds per scanning position in their scanner coordinate system (i.e. scanning position is equal to origin of coordinate system) are used for data processing. In addition, the transformation matrices from the registration process are necessary.

A major advance of the Poisson based triangulation is its ability to achieve homogeneously shaped triangles with vertices, following an almost regular distribution. This is caused by the voxel based evaluation of the scalar field. In the following, we use the term resolution as an acronym for the resolution of the voxel structure. Assuming $3 \mathrm{~mm}$ point density per scan and, for most regions, approximately three overlapping scans would support a resolution of approximately $5 \mathrm{~mm}$. Although we split the objects into three groups, this resulted in triangulations with about 100 million triangles each. Considering the visualization capabilities, we decided to reduce the resolution to $10 \mathrm{~mm}$ during processing. Consequently, the triangulation model shown in Figure 5 consists of three tiles (left, right, Neptune including rocky grotto) with altogether 120 million triangles including the main basin.

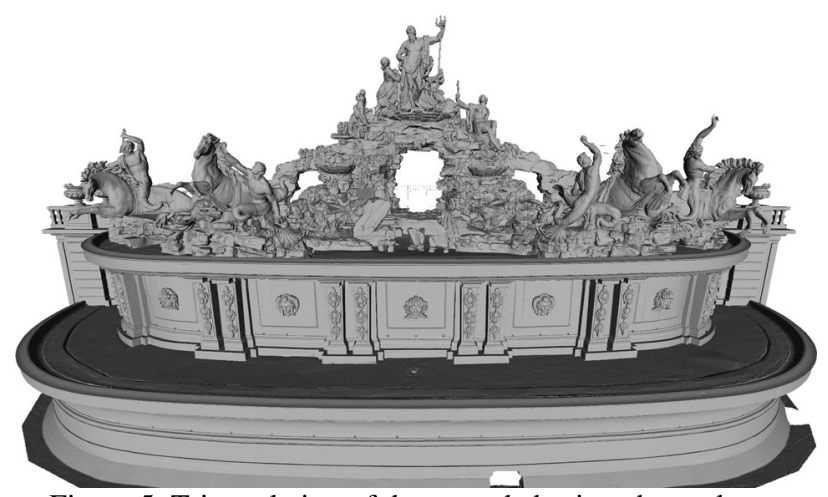

Figure 5. Triangulation of the cascade basins, the sculpture ensembles and the rocky grotto with Neptune on top of it.

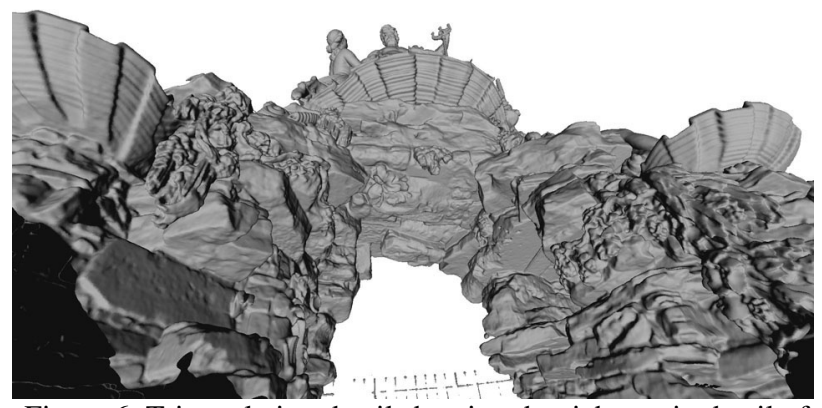

Figure 6. Triangulation detail showing the richness in detail of the model.

\subsection{Visualization}

While accurate documentation requires a high resolution of the triangulation, for visualization purposes, the number of polygonal faces (i.e. triangles) has to be reduced by one or two orders of magnitude, or even more. To ensure a photorealistic appearance, displacement mapping is used. Figure 7 shows a normal map (left) and a rendering (right) of the wall of the cascade basin of the Neptune Fountain, where a planar surface was used as geometrical foundation. The normal map was automatically generated from the laser scanning data.
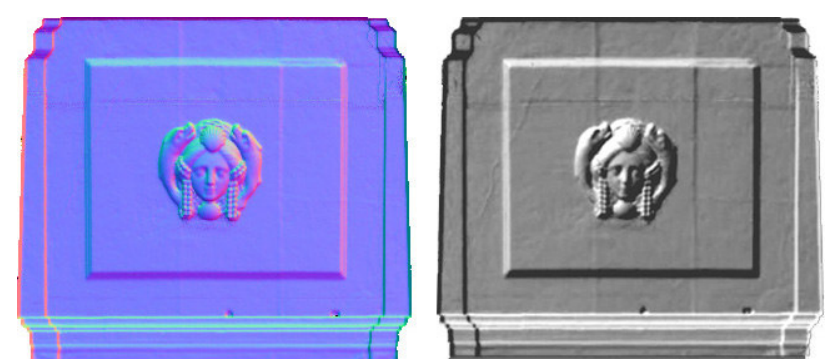

Figure 7. Displacement mapping to reduce the number of polygons. Left: Normal map, Right: Rendering

Additionally, the entire object has to be split into developable sub-objects. Subsequently, interactive texture mapping using real textures is possible. Figure 8 shows a textured model of the sculpture group arranged around the Neptune sculpture on top of the rocky grotto. The visualization model has been prepared for proper visualization on tablet PCs and smart phones. A high-resolution rendering of the entire scene including a textured digital terrain model of the surrounding park is shown in Figure 9. 


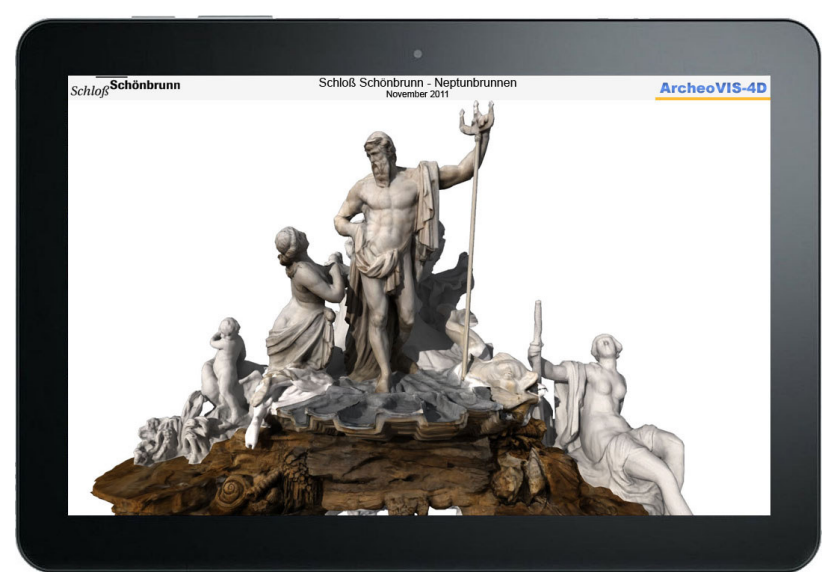

Figure 8. 3D rendering on tablet PC using image texture, bump mapping and artificial lightning.

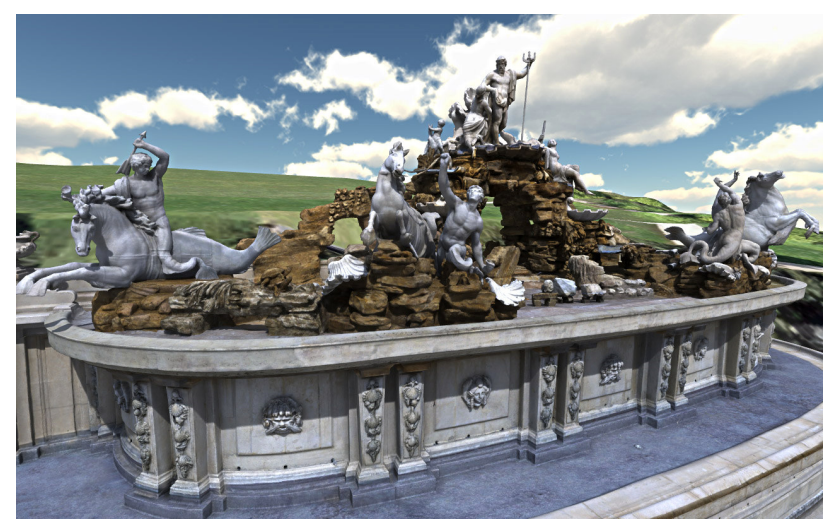

Figure 9. High-resolution 3D real-time rendering of the entire scene.

\section{DISCUSSION}

For a documentation model, the achievable accuracy as well as the richness in detail represented is of major importance. According to the technical specification (www.faro.com), the Faro Photon 120 has a distance measurement precision (i.e. repeatability) of approximately $1 \mathrm{~mm}( \pm 0.4 \mathrm{~mm}$, dependent on the reflectivity of the object) at a measurement distance of 10 meter. The overall accuracy accumulating systematic errors in addition to the noise is $2 \mathrm{~mm}$ at a measurement distance of 25 $\mathrm{m}$. To investigate the achieved accuracy of the model, we used two measures. The first is implicitly determined. The Poisson triangulation applied determines the implicitly determined surface, described by the scalar field defined by the given pointcloud (confer section 2). If the discrepancies between overlapping scans exceed a certain distance, the resulting triangulation has detectable deficiencies in the respective regions. The reason for this is that the voxel density of the octree used to derive the triangulation is lower in regions without actual measurement points. In our case, discrepancies would result in an improper triangulation. Second, we analysed the discrepancies between individual point-clouds with respect to the resulting triangulation model. Considering the instrument's accuracy of $2 \mathrm{~mm}$, we assume that each pointcloud has an inner accuracy of this magnitude. However, additional, systematic errors decrease the achievable accuracy. Those systematic errors are introduced by the individual processing steps, hence comprising remaining discrepancies of the registration as well as deformations of the Poisson approach (Nothegger, 2011). Figure 10 shows the discrepancies between a single point-cloud (scanning position 6) with respect to the final documentation model. For most regions, the discrepancies show a magnitude smaller than $+/-5 \mathrm{~mm}$, i.e. the achieved accuracy. In addition, Figure 10 proofs the stability of the scaffold used. If the stability of the scaffold would not have been sufficient, a "wavy" pattern would occur in the differences.

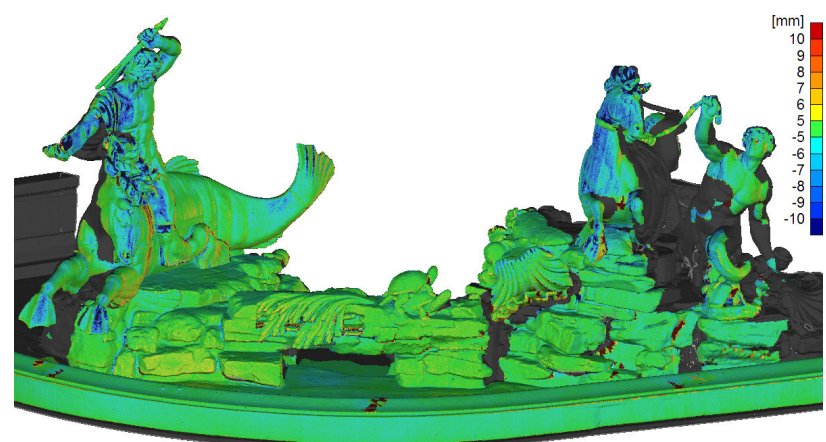

Figure 10. Discrepancies between a single point-cloud (scanning position 6) and the final documentation model.
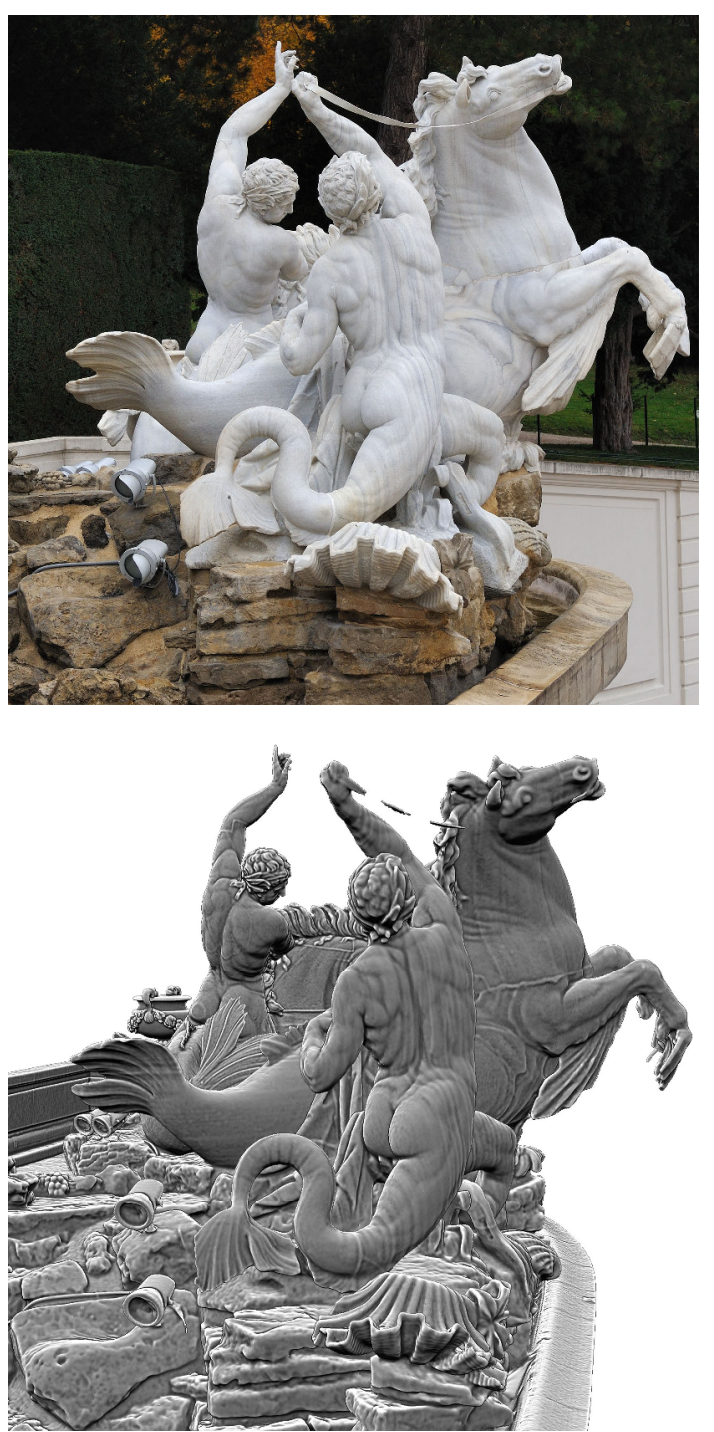

Figure 11. Influence of translucent material (i.e. marble) on the achievable accuracy of the TLS measurements. Upper: Marble sculpture with typical linear pattern. Lower: Triangulation model showing the same linear structures at the marble objects

(i.e. Triton) while the sandstone is modelled properly. 
TLS is based on an emitted laser beam (either pulsed or amplitude modulated continuous wave) to determine the distance between the scanner and the object where the signal is reflected. If the material of the object is translucent at the wavelength used for the scanning instrument (typically visible or near infrared), the energy is partly reflected on the surface structure, but at least a fraction of the energy interacts with the subsurface structure. Dorninger \& Nothegger (2011) described this phenomenon and investigated the correlation of the visible appearance of crystalline marble stones and the distance measurement error. The testing objects showed maximum errors of up to $20 \mathrm{~mm}$. El-Hakim et al. (2008) and Godin et al. (2001) describe the same phenomenon.

Figure 11 upper shows one of the five ensembles of sculptures on top of the cascade basins. Especially one of the Tritons (a creature half-man and half-fish) has a typical stripe pattern on his back and his leg. Figure 11 lower shows the triangulation mesh of this object. The linear structures are clearly visible as well. In addition, the splices between the individual blocks building up the horse can be seen as raised structures within the triangulation model while those are engraved in reality. We determined the discrepancies of the real surface with respect to the triangulation model by means of reference surfaces and in general, the maximum discrepancies are some $8 \mathrm{~mm}$. The same magnitude can be seen in Figure 10. The blue areas occur in orthogonal direction with respect to the scanning position. Hence, the highest energy is emitted to those regions causing the maximum of distance measurement errors there.

\section{CONCLUSIONS}

We demonstrated a highly automated workflow for 3D model generation of huge objects for documentation purposes. For economic reasons, we propose the application of phase-shift laser scanner for the data acquisition. As demonstrated, the achievable accuracy exceeds that of image based technologies. In comparison to close-range scanners which allow for a higher accuracy, both data acquisition and model generation is possible at significantly lower costs, especially for huge objects.

The presented 3D triangulation model was computed using an implementation of the Poisson triangulation approach. In comparison to explicit surface definition, the thus determined triangulation has fewer deficiencies considering twisted triangles and data gaps. This reduced the required interactive post-processing significantly.

The absolute accuracy, highly correlated to the accuracy of the reference point network, was $5 \mathrm{~mm}$. Investigating local richness in detail as well as local accuracy investigation by means of difference analysis of overlapping scans have shown, that locally the achieved accuracy is even better.

The issue of translucent material remains unsolved. While the scanners used have no problem if scanning opaque materials like sandstone or lime stone, there are significant deficiencies in the data if translucent materials like marble are scanned. For the marble sculptures of the Neptune Fountain, the maximum discrepancies observed have a magnitude of up to $8 \mathrm{~mm}$. This is not sufficient; however it is less compared to results of former experiments as discussed.

The problem concerning translucent materials is subject of current investigation. However, it has been demonstrated, that using phase-shift scanners makes it economically feasible to document huge objects with a highly detailed surface structure. Current instruments are small and lightweight and due to their high measurement rates of up to 1 million points per second, very fast data acquisition is possible. In addition, the presented highly automated processing chain enables the generation of highly detailed 3D models. The achievable resolution and implicitly the achievable accuracy are limited more by the representation medium than by the data acquisition instrument and the applied modelling approach.

As demonstrated, the high resolution models can be applied for both highly detailed documentation and for visualization. If data reduction is properly applied, the models are applicable even for presentation on smart devices.

\section{REFERENCES}

Akca, D., Gruen, A., 2007: Generalized Least Squares Multiple 3D Surface Matching. The International Archives of the Photogrammetry, Remote Sensing and Spatial Information Sciences, Vol. 36, 3/W52.

Akca, D. (2012). 3D Modeling of cultural heritage objects with a structured light system. Mediterranean Archaeology and Archaeometry, 12(1), pp. 139-152.

Amenta, N., Choi, S., Kolluri, R.K., 2001. The Power Crust. In: Proceedings of the $6^{\text {th }}$ ACM symposium on solid modeling and applications, Ann Arbor, Michigan, United States: ACM, pp. 249-266.

Bolitho, M., Kazhdan, M.M., Burns, R.C., Hoppe, H., 2009. Parallel Poisson Surface Reconstruction, ISVC (1), G. Bebis, R.D. Boyle, B. Parvin, D. Koracin, Y. Kuno, J. Wang, R. Pajarola, P. Lindstrom, A. Hinkenjann, M.L. Encarnação, C.T. Silva, and D.S. Coming, Eds., Springer, pp. 678-689.

Curless, B., Levoy, M., 1996. A Volumetric Method for Building Complex Models from Range Images. In: Proceedings of the $23^{\text {rd }}$ annual conference on Computer graphics and interactive techniques, ACM, pp. 303-312.

Deseilligny, M.P., Clery, I., 2011. Apero, an open source bundle adjusment software for automatic calibration and orientation of set of images. In: Proceedings of the ISPRS Symposium, 3DARCH11, pp. 269-277.

Dorninger, P., Nothegger. C., 2010. Das Messverhalten Terrestrischer Laser Scanner an Steinoberflächen, In: Publikationen der Deutschen Gesellschaft für Photogrammetrie, Fernerkundung und Geoinformation e.V., Band 19, pp. 504-512.

Edelsbrunner, H., Mücke, E.P., 1994. Three-dimensional Alpha Shapes, 1994.

El-Hakim, S., Beraldin, J.-A., Picard, M., Cournoyer, L, 2008. Surface Reconstruction of Large Complex Structures from Mixed Range Data - The Erechtheion Experience. In: International Archives of the Photogrammetry, Remote Sensing and Spatial Information Sciences, 20(B5).

Godin, G., Rioux, M., Beraldin, J.-A., Levoy, M., Cournoyer, L., Blais, F., 2001. An Assessment of Laser Range Measurement on Marble Surfaces. In: Proceedings of the $5^{\text {th }}$ 
Conference on Optical 3D Measurement Techniques, October 1-4, 2001, Vienna, Austria.

Golparvar-Fard, M., Bohn, J., Teizer, J., Savarese, S., \& PenaMora, F., 2011. Evaluation of image-based modeling and laser scanning accuracy for emerging automated performance monitoring techniques. Automation in Construction, 20(8), pp. 1143-1155.

Hoppe, H., 1994. Surface Reconstruction from Unorganized Points, PhD Thesis, University of Washington.

Kazhdan, M., Bolitho, M., Hoppe, H., 2006. Poisson surface reconstruction, In: Proceedings of the fourth Eurographics symposium on Geometry processing, Eurographics Association Aire-la-Ville, Switzerland, pp. 61-70.

Kazhdan, M., Hoppe, H., 2013. Screened Poisson Surface Reconstruction, Transactions on Graphics, TBA 2013.

Nothegger, C., 2011. Improving Completeness of Geometric Models From Terrestrial Laser Scanning Data, In: Proceedings of the $23^{\text {rd }}$ CIPA Symposium, Prague, Czech Republic.

Pierrot-Deseilligny, M., De Luca, L., \& Remondino, F., 2011. Automated image-based procedures for accurate artifacts $3 \mathrm{D}$ modeling and orthoimage generation. In: Proceedings of the $23^{\text {rd }}$ CIPA Symposium, Prague, Czech Republic.

Rusinkiewicz, S., Levoy, M., 2001. Efficient variants of the ICP algorithm. In: 3-D Digital Imaging and Modeling, Third International Conference on 3-D Digital Imaging and Modeling, IEEE, pp. 145-152.

Segal, A., Haehnel, D., Thrun, S., 2009. Generalized-ICP. In Robotics: Science and Systems, (2).

Ullrich, A., Schwarz, R., Kager, H., 2003. Using hybrid multistation adjustment for an integrated camera laser-scanner system. In: A. Gruen and H. Kahmen (eds), Optical 3-D Measurement Techniques: Proceedings of $6^{\text {th }}$ conference on optical 3-D Measurement Techniques, Zurich, pp. 298-305.

Vergauwen, M., Van Gool, L., 2006. Web-Based 3D Reconstruction Service. Machine Vision Applications 17, pp. 411-426.

\section{ACKNOWLEDGEMENTS}

The development of the triangulation method used was funded by the Österreichische Forschungsförderungsgesellschaft (FFG) within the project Hybrid3D (Pr.Nr. 829554) and supported by $4 D-I T G m b H$ (www.4d-it.com). 